\title{
Manejo nutricional de la glucogenosis
}

\author{
M.C. Carlos Castillo D. ${ }^{1,2}$; M.C. Francisco Lagrutta $S^{3}$ \\ Nutritional management in glycogenosis
}

Three cases of glycogenosis are presented. They were recognized by clinical evidence and hepatic biopsy specimens. Dietary treatment with a normocaloric diet and raw corn starch every 6 hours was given. In cases 1 and 2 hepatic size returned to normal and they showed an initial catch up growth without signs of renal disease or other complications. Case 3 showed persistent hepatomegaly, normal growth pattern and no hypoglicemic episodes independently of nutritional management. These cases represent our initial experience on nutritional intervention in glycogenosis, even though enzymatic tests were not done, which are considered essential for optimal diagnosis and nutritional therapy of gly cogen storage disease.

(Key words: glycogenosis, gly cogen storage disease, diet therapy, corn starch.)

Las glucogenosis son un conjunto de enfermedades hereditarias que se caracterizan por ausencia o deficiencia de la actividad de diversas enzimas que participan en el metabolismo del glucógeno y que causan su depósito en diversos órganos ${ }^{1}$. La forma más frecuente de ellas parece ser la tipo IA o enfermedad de Von Gierke; sin embargo, en los casos publicados en el pais, la forma diagnosticada con más frecuencia ha sido el tipo $\mathrm{III}^{2}$. Sus manifestaciones están mediadas principalmente por hipoglicemia, pudiendo llevar a convulsiones, daño neurológico, retraso severo de talla, hepatomegalia, acidosis láctica, hiperuricemia e hiperlipidemia ${ }^{3}$. Por este motivo se han propuesto diversas terapias nutricionales que tienden a mantener glicemia normal, tales como: dietas fraccionadas a través del día ${ }^{4}$, alimentación parenteral ${ }^{5}$, alimentación enteral nocturna ${ }^{6}$ e incluso derivaciones porto-cava ${ }^{7}$.

El propósito de esta comunicación es mostrar nuestra experiencia inicial en el tratamiento de las glucogenosis mediante una dieta en base a almidón de maíz crudo (maicena), propuesto por otros investigadores en los últimos años ${ }^{3, B}$.

\section{CASOS CLINICOS}

1. Varón de 5 años y 10 meses de edad en la actualidad. Pesa y talla de nacimiento normales $(3.850 \mathrm{~g}$

1. Profesor Asistente Instituto de Nutrición y Tecnología de los Alimentos, Universidad de Chile.

2. Unidad de Nutrición, Servicio de Pediatría, Hospital Sótero del Río.

3. Médico Pediatría Hospital del Niño, Panamá, República de Panamá. y $51,5 \mathrm{~cm}$, respectivamente) con evolución pondoestatural adecuada hasta los 18 meses. A los 21 meses se comprobó menor velocidad de crecimiento y hepatomegalia que no fueron consignadas nuevamente sino hasta los 28 meses. A esta edad la carotinemia exa normal, D-xilosa sanguínea postingestión de dosis estándar $44 \mathrm{ug} / \mathrm{dl}$, edad ósea cotrespondiente a 18 meses, glicemia $60 \mathrm{mg} \%$, protrombinemia normal, transaminasas $70 \mathrm{U}$ (GPT y GOT), ceruloplasmina $31 \mathrm{mg} / \mathrm{dl}$, albuminemia $4,5 \mathrm{~g} / \mathrm{dl}$ y antitripsina $1,39 \mathrm{~g} / \mathrm{d}$. En la biopsia hepática, tinción PAS, se observó abundante depósito citoplasmático de glucógeno $y$ células agrandadas de núcleo central, sin atras alteraciones, confirmando el diagnóstico de glucogenosis. Se indicó alimentación fraccionada por sonda nasogástrica durante la noche, peso cumplió mal el tratamjento y el tamar̂o del hígado se mantuvo, desde los 2 años 10 meses de edad, $8 \mathrm{~cm}$ bajo el reborde, con $12 \mathrm{~cm}$ de proyección en la linea medioclavicular; el desarrollo psicomotor ptosiguió normalmente y no presentó convulsiones u otros síntomas de hipoglicemiz. Sin embargo, a los 4 años 2 meses las glicemias seriadas mostraban las concentraciones más bajas a las 8 horas $(68 \mathrm{mg} / \mathrm{dl})$. sin llegar a hipoglicemia y concentraciones elevadas de lactato sérico en ayunas $(\mathbf{3 4 , 3} \mathrm{mg} / \mathrm{dl}, \mathrm{VN} 5,7-22 \mathrm{mg} / \mathrm{dl})$. Se intició entonces terapia dietética con maicena cruda en forma de papilla $(1,5 \mathrm{~g} \cdot \mathrm{kg}$ - dosis cada 6 horas $)$, se restringió el aporte total de hidratos de carbono a $55 \%$ de las calorías totales, suministrando el resto como proteínas (10\%) y lipidos (35\%), en 5 horarios diurnos (desayuno, colación, almuerzo, once y comida). Presentó leve diarrea $y$ dolor abdominal en las primeras dos semanas, que cedieron después. Las glicemias basales aumentaron a 80 y $85 \mathrm{mg} / \mathrm{dl}$. Un mes y medio después de injciar este régimen de alimentación se apreció disminución de la hepatomegalia a $4,5 \mathrm{~cm}$ bajo el reborde y aumento de la velocidad de crecimiento en estatura $(2,5 \mathrm{~cm}$ en el mismo tiempo). Cuatro meses después de inicjada la terapia, el hígado estaba $1 \mathrm{~cm}$ bajo el reborde costal y seguja con buena velocidad de crecimiento, acercándose su talla al percentil 5 para la edad (figura). Estas tendencias se mantienen 2 años 


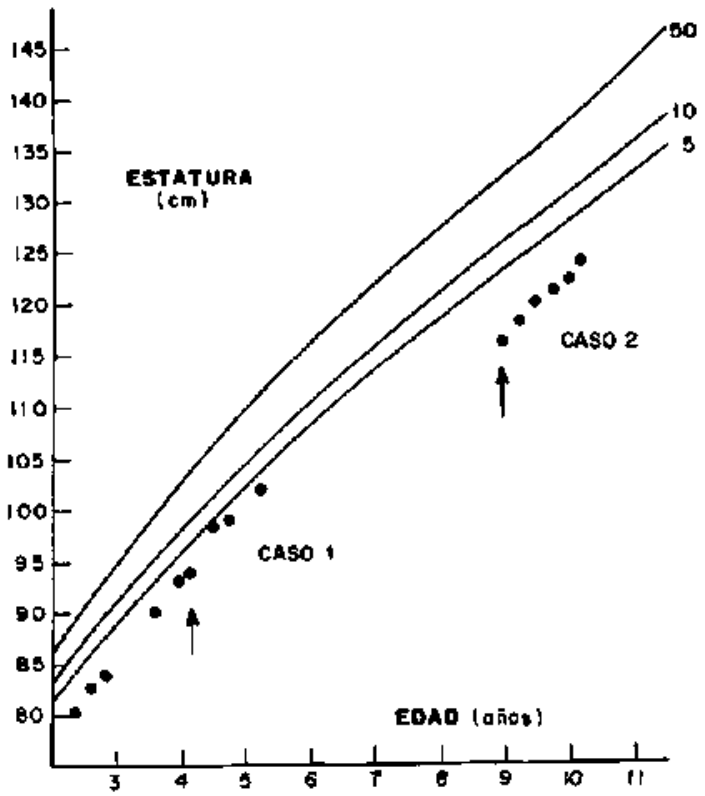

Figura: Evolución de la estatura en los pacientes 1 y 2 desce la introducción de harisa cruda de maíz (flecha).

después de iniciađo el tratamiento, con buen cumplimiento del mismo y sin signos de compromiso renal.

2. Nifla de 10 años 2 meses de edad, hospitalizada en noviembre de 1987 en el Servicio de Pediatria del Hospital Sótero del Rio por retraso de estatura y hepatomegalia de 6 a $7 \mathrm{~cm}$ bajo el reborde costal, proyección hepática total de $12 \mathrm{~cm}$ en la línea medioclavicular, Presentaba crisis de fatiga cada 15 a 20 días, que mejoraban empíricamente con glucosa oral. Además, había sufrido convulsiones a los 3 años de edad, que fueton controladas en otro hospital sin diagnóstico definitivo. El electroencefalograma era normal. La biopsia hepática al ingreso demostró arquitectura lobulillar conservada, abundante depósito de glucógeno citoplasmático con tinción de PAS $y$ hepatocitos aumentados de tamario. Las determinaciones seriadas de glicemia a través del día mostraron cifras levemente bajas en ayunas, a las 8 horas ( $46 \mathrm{mg} \%$ ) y concentraciones sanguíneas de lactato de 7,2 y $8,9 \mathrm{mg} / \mathrm{dl}$ a las 8 y 1 horas, respectivamente (VN $5,7-22 \mathrm{mg} / \mathrm{dl}$ ), uricemia $6.4 \mathrm{mg} \%$, levemente elevada, transaminasa SGOT $197 \mathrm{U}$. A las $24 \mathrm{~h}$ de iniciado el tratamiento con maicena cruda en papilla acuosa $1,5 \mathrm{~g} \cdot \mathrm{kg} \cdot$ dosis cada 6 horas, se mantenian los niveles de glicemia y habían aumentado los de lactato sético a las 2 AM $(32,6 \mathrm{mg})$ dl). Egresó con dicho tratamiento y una dieta normocalótica con las mismas proporciones de nutrientes que en el caso 1. Durante su evolución tendió a presentar disminución de la hepatomegalia, llegando ésta, $6 \mathrm{me}$ ses después del alta, a $3 \mathrm{~cm}$ bajo el reborde en la línea medioclavicular y mostrando incremento estatural de $4 \mathrm{~cm}$ en el mismo periodo de tiempo. Por incumplimiento del régimen suftió una recaída a los 10 meses de prescribir el tratamiento, llegando la hepatomegalia a $8,5 \mathrm{~cm}$ bajo el reborde con detención de la talla. A continuación reinició el tratarniento y 3 meses después el hígado se palpaba tan sólo a $1 \mathrm{~cm}$ bajo el reborde en línea medjoclavicular, mientras se registraba un incremento de $3 \mathrm{~cm}$ en la talla de los últimos 4 meses del año (figura 1), lo que evidenciaba tendencias a la recuperación de estatura. No presenta signos de desarrollo puberal.

3. Lactante de 20 meses de edad, de sexo masculino, admitido en el Hospital Sótero del Río en junio de 1988 por hepatomegalia de $9 \mathrm{~cm}$ bajo el reborde costal. En la ecografía la proyección hepática era de $14 \mathrm{~cm}$ en línea medioclavicular. Antecedentes de lactancia natural prolongada y soplo sistólico H/IV irradiado a cuello. Durante su hospitalización no se comprobó hipoglicemia, alteraciones en el lactato sérico, ni compromiso pondoestatural, La biopsia hepática mostró arquitectura lobulillar conservada, abundante depósito de glu. cógeno citoplasmático con tinción PAS, núcleo central pequefio y células hepáticas agrandadas. Se inició tratamiento con maicena cruda y una dieta de iguales características que en los dos casos anteriores. Luego de 4 meses de tratamiento (octubre 88) no había disminuido la hepatomegalia, persistiendo el hígado a $9 \mathrm{~cm}$ bajo el reborde costal. Se pensó en la posibjlidad de formas menos frecuentes de glucogenosis. Se ordenaron ultrasonografías cardíaca y renal, que resultaron normates, y se inicló en octubre dleta normocalótica sin maicena; sin cambios en la hepatomegalia hasta diciembre 88. Su crecimiento pondoestatural se mantuvo normal. Como en los casos anteriores, no ha sido posible efectuar estudio enzimático para establecer la forma de glucogenosis.

\section{DISCUSION}

Una de las díficultades en el manejo de este tipo de pacientes es el diagnóstico preciso. Aún no se dispone en forma habitual en nuestro país de las pruebas enzimáticas que permiten distinguir las diversas formas de glucogenosis, siendo su uso más bien esporádico, por lo que se emplean diversas pruebas metabólicas para hacer una aproximación. En el estudio de Brinck y cols., del Hospital Luis Calvo Mackenna ${ }^{2}$, los 22 casos diagnosticados en 15 af́os, fueron estudiados básicamente mediante biopsia hepática, pruebas de tolerancia a la glucosa y al glucagón, concentra. ciones de lactato sérico, uricemia, características clínicas y, algunos, con mediciones histoquímicas de glucosa-6-fosfatasa hepática. En los pacientes estudiados por nosotros, además de los aspectos clínicos, sólo fue posible efectuar mediciones seriadas de glicemia, lactato sérico, cuerpos cetónicos en sangre, biopsia hepática, lo que no permite identificar con más precisión el tipo de glucogenosis. De todos modos los datos disponibles hacen sospechar que los primeros 
dos casos corresponden a glucogenosis tjpo I o IL y las limitaciones señaladas no invalidan la prue. ba terapéutica.

En el primer caso la respuesta a la terapia fue muy adecuada, favorecida por un nivel educacional bueno de la familia, que ha pernitido buena adhesión al tratamiento $\mathrm{y}$ adquisición oportuna de los componentes dietéticos. En el segundo caso esto no ocurrió, debido a causas económicas, pero con apoyo de un plan educativo ha sido posible el cumplimiento de la dieta, con buena respuesta del crecimiento y reducción de la hepa. tomegalia. Afortunadamente, ambos casos no son de las formas más severas, lo que ha permitido lograr un equilibrio metabólico relativamente rápido. La falta de respuesta en el tercer paciente hace resaltar la necesidad de contar con medios adecuados para hacer el diagnóstico preciso de la falla enzimática correspondiente, pues es posible que este niño presente otra forma de glucogenosis, alguna de las que no responden a este tratamiento $y$ pueden tener una evolución más severa. Se están intentando en la actualidad otras formas de tratamiento nutricional con este paciente.

Aunque la glucogenosis es relativamente poco frecuente, sus consecuencias justifican los esfuerzos por ofrecer a los pacientes un manejo paliativo. En el caso de la glucogenosis tipo LA, a los síntomas mencionados, que a veces pueden ser poco acentuados, hay que agregar la posibilidad, descrita recientemente, de enfermedad renal, a largo plazo ${ }^{9}$. En efecto, el seguimiento de estos pacientes hasta adultos ha permitido constatar que, al agrandamiento renal comprobable en la edad pediátrica, se pueden agregar, con alta probabilidad, signos de enfermedad renal, tales como hipertensión, proteinuria, hematuria y disminución del aclaramiento de creatinima; estas alteraciones pueden llegar a insuficiencia renal crónica y muerte. No está claro aún si el control dietético de la glicemia en el rango normal puede disminuir este alto riesgo, pero es probable que ello se conozca en el futuro, lo que es un motivo aún mayor para intentar el manejo dietético adecuado de la glucogenosis. Ninguno de nuestros pacientes presenta complicaciones renales hasta el momento.

De las terapias usadas previamente, la alimentación parenteral o enteral nasogástrica con infusión nocturna continua pueden ser efectivas. $S$ in embargo, no es factible el empleo pro- longado de la primera y es difícil lograr buen cumplimiento de la segunda. En el caso 1 se intentó la infusión enteral nocturna, pero no se logró buen cumplimiento a pesar de las condiciones favorables del medio familiar.

La terapia dietética con almidón de maíz crudo ha sido propuesta recientemente ante la comprobación de que es capaz de mantener glicemias normales en forma permanente $y$ pro. longada ${ }^{3,8}$. Aunque puece también provocar algunas dificultades en la adhesión al tratamiento, por el costo del producto, la necesidad de un horario nocturno de alimentación $y$ la aceptabilidad del alimento cnudo, ellas son mucho menores que en los otros tratamientos. Tampoco ha sido problema la aparición, en algunos casos, de diarreas o dolor abdominal en los primeros días, ya que ceden posteriormente.

Este método de manejo retardaría el vacia. miento gâstrico, produciendo liberación sostenida y prolongada de su contenido hacia el intestino, en consecuencia elevaciones postpran. diales menos intensas de la glicemia y nomoglicemias más duraderas, hasta la siguiente alimentación ${ }^{10}$. Junto a esta mejor regulación metabólica se observa normalización de lactacidemia, disminución del depósito hepático del glucógeno, nomalización del tamaño del hígado y mejoría en la velocidad de crecimiento; también hemos observado una disminución de la facie "regordeta" que se ha mencionado como habitual en esta forma de glucogenosis.

Chen y cols. ${ }^{3}$ en 1984 propusieron el esque. ma actualmente en estudio, validando el uso de almidón de maíz crudo, en forma de papilla oral cada 6 horas. Ellos demostraron una tendencia adecuada a la nomoglicemia en el transcurso del dia y la noche, comparable con la producida con la administración de fórmulas en infusión nasogástrica nocturna asociada con alimentación fraccionada diurna.

Cabe preguntarse si es indispensable usar almidón de maíz crudo ${ }^{11,12}$. Estudios efectuados con almidones de papas, arroz o porotos cocidos han mostrado estrecha correlación negativa entre el tiempo de vaciamiento gástrico $y$ las respuestas glicémica y de insulina ante la ingestión de papas o arroz en una dieta mixta, siendo menor la elevación de la glicemia con el arroz ${ }^{13}$. En estudios efectuados en adultos sanos se ha encontrado menor respuesta de insulina y gli. cemia, mientras mayor es el tamaño de partícula de trigo, maíz o avena. Assimismo, se observó 
que una comida en base a avena tendía a provocar menor respuesta en la insulinemia y la glicemia que las basadas en trigo o maíz ${ }^{10}$. Aunque dichos alimentos se emplearon cocinados y en sujetos adultos normales, estos resultados pueden ser un avance digno de estudiar en nitios con glucogenosis. También se ha mostrado la utilidad de hidratos de carbono de digestión lenta, como el propuesto almidón de maíz crudo, y comprobado la posibilidad de usar otros hidratos de carbono de digestión semilenta o lenta como la cebada o el couscous ${ }^{14}$. Aunque esta forma de manejo parece muy adecuada para la glucogenosis tipo IA, recientemente han aparecido algunas comunicaciones preliminares que también muestran la efectividad del almidón de maiz crudo en la glucogenosis tipo III (ausencia de la enzima destamificadora amiloalfa 1,6 -glucosidasa $)^{15}$ y mejoría parcial en la glucogenosis tipo IV (déficit de la enzima ramificadora alfa 1,4-glican 6-glicosiltransferasa) ${ }^{16}$.

En conclusión, podemos decir que el esquema dietético basado en almidón de maíz crudo y restricción leve del aporte total de hidratos de carbono ofrece una alternativa práctica de manejo a los pacientes con glucogenosis. Se hace necesario contar en nuestro país con métodos de diagnóstico enzimático de las diferentes formas de la enfermedad, que permitirian definir mejores estrategias de tratamiento en estos ca. sos. Asimismo, el diagnóstico más precoz de la glucogenosis, unido a este tipo de terapias nutricionales, presumiblemente disminuirá el riesgo de complicaciones agudas y en el largo plazo.

\section{RESUMEN}

Se presentan 3 casos de glucogenosis comprobada clínicamente y con biopsia hepática, en los cuales se realizó tratamiento con almidón de maíz crudo (maicena). En los casos 1 y 2 ha disminuido la hepatomegalia, el incremento ponderal es adecuado y no presentan signos de faila renal u otras complicaciones. En el caso 3, persiste la hepatomegalia, pero el incremento pondoestatural es adecuado; esto último aparentemente no guaría relación, en este caso, con el tratamiento dietético. Esta es una experiencia inicial en el manejo nutricional de casos de glucogenosis. Es indispensable incorporar el estudio enzimático para definir con mayor exactitud el diagnóstico y poder dirigir más adecuadamente las modificaciones en la terapia nutricional.

(Palabras clave: glucogenosis, tratamiento dietético, hipoglicemia, almidón de maíz.)

\section{REFERENCIAS}

1. Hug $G_{*}$ : Defects in metabolism of carbohydrates. En: Textbook of Pediatrics. Nelson W.E., Behrman R.E., Vaughan V.C. (eds.). W.B. Saunders Company, Philadelphia, USA 1983, pp. 455-465.

2. Brinck P., Olguin $H$., Zacarias J.: Glucogenosis. Hallazgos clínicos y de laboratorio en 22 enfermos. Rev Chil Pediatr $1985 ; 56: 403-411$.

3. Chen Y.T., Camblath M. Sidbury J.B.: Comstarch therapy in type I glycogen-storage disease. N Eng J Med 1984; 310: 171-175.

4. Leonard J.Y., Dunger D.B.: Hypoglycemia complicating feeding regimen for glycogen-storage disease. Lancet 1978; 2: 1203-1204.

5. Folkman J., Phillippart A., Tze W.J., Crigler J., Jr.: Portocaval shunt for glycogen storage disease: value of prolonged intravenous hyperalimentation before surgery. Surg 1972;72:306-314.

6. Greene H.L., Slonim A.E., O'Nell J.A. Ir., Burr I.M.: Continuous nocturnal intragastric feeding for management of Type I glycogen-storage disease. N Engl J Med 1976; 294: 423-425.

7. Ridall A.G., Davies R.P., Clark A.D.: Portocaval transposition in the treatment of glycogen storage disease. Lancet $1966 ; 2 \div 1146-1148$.

8. Smit G.P.A., Berger R., Potasnick R., Moses S.W., Fernandes $J$.: The dietary treatment of children with type I glycogen storage disease with slow release carbohydrate. Pediatr Res 1984; 18: 879881.

9. Chen Y.T., Coleman R.A., Scheinman J.I. Kolbeck P.C., Sidbury J.B.: Renal disease in type I glycogen storage disease. N Engl J Med 1988; 318: 7-11.

10. Heaton K.W., Marcus S.N., Emmett P.M. Bolton C.H.: Particle size of wheat, maize and oat test meals: Effects on plasma glucose and insulin responses and on the rate of starch digestion in vitro. Am J Clin Nutr $1988 ; 47: 675-682$.

11. Snow P., $O^{\prime}$ Dez $K$. Factors affecting the rate of hydrolysis of starch in food. Am $J$ Clin Nutr $1981 ; 34: 2721 \cdot 2727$.

12. Collings P., Willams $P$, MacDonald I.: Effects of cooking on serum glucose and insulin responses to starch. Br Med J 1981;282: 1032.

13. Tordotir J., Alpsten M., Anderson D., Brummer R.J.M., Anderson $H$.: Effect of different starchy foods in composite meals on gastric emptying rate and glucose metabolism I. Comparisons between potatoes, rice and white beans. Hum Nutr: Clin Nutr 1984; 38C: $329-338$.

14. Smit G.P.A., Ververs M.T. Belderot B., Van Rijn M., Femondes J.: Complex carbohydrates in the dietary management of patients with glycogenosis caused by glucose-6-phosphatase deficiency. Am J Clin Nutr 1988; 48: 95-97. 
15. Borowitz S.M., Greene H.L: Case report. Cornstarch therapy in a patient with type III glycogen storage disease. J Pediatr Gastroenterol Nutr 1987; 6: 631-634.
16. Greene H.L., Ghishan F.K., Brown B., McClenathan $D . Y$. Freese $D$.: Hypoglycemia in type IV glycogenosis: Hepatic improvement in two patients with nutritional management. J Pediatr 1988; 112: 55-58. 